\title{
Germanica
}

\section{LTI. Caractéristiques linguistiques d'un langage « inhumain »}

In lingua veritas

LTI. Sprachliche Merkmale einer "unmenschlichen» Sprache

\section{Nicole Fernandez Bravo}

\section{OpenEdition}

\section{Journals}

Édition électronique

URL : http://journals.openedition.org/germanica/2464

DOI : 10.4000/germanica.2464

ISSN : 2107-0784

Éditeur

Université de Lille

\section{Édition imprimée}

Date de publication : 30 décembre 2000

Pagination : 147-174

ISBN : 9782913857094

ISSN : 0984-2632

Référence électronique

Nicole Fernandez Bravo, «LTI. Caractéristiques linguistiques d'un langage « inhumain » ", Germanica [En ligne], 27 | 2000, mis en ligne le 01 avril 2014, consulté le 06 octobre 2020. URL : http:// journals.openedition.org/germanica/2464; DOI : https://doi.org/10.4000/germanica.2464

Ce document a été généré automatiquement le 6 octobre 2020.

(c) Tous droits réservés 


\section{LTI. Caractéristiques linguistiques d'un langage « inhumain »}

In lingua veritas

LTI. Sprachliche Merkmale einer « unmenschlichen» Sprache

Nicole Fernandez Bravo

\section{Introduction}

1 LTI, la Langue du troisième Reich, la langue du nazisme apparaît comme le point nodal commun à deux ouvrages de Klemperer, le journal intime rédigé durant le fascisme, carnet de notes d'un " philologue » de terrain qui découvre le langage de propagande et ses ravages sur le peuple et la culture allemandes, et l'ouvrage dont il constitue le titre, LTI. Notizbuch eines Philologen (1947). Malgré son sous-titre, il ne s'agit pas d'un livre de linguiste, au sens où l'on entend aujourd'hui ce terme, tant il témoigne de l'intrication du langage avec le vécu de Klemperer, rendant la distance et la rigueur scientifiques impossibles. LTI s'ouvre ainsi par un Vorspiel et mêle des considérations philologiques sur des occurrences de vocabulaire frappantes notées dans le journal, avec des anecdotes et des souvenirs du proche passé. La dimension temporelle et historique est ce qui donne son unité à ce qui apparaît à la lecture du sommaire comme composite, un mélange d'«expressions » (« Wejen Ausdrücken »), comme l'indique le titre de l'épilogue, d'évocations de brefs moments narrés (ch. XVI : An einem einzigen Arbeitstag) ou de périodes plus larges de l'histoire (ch. VIII: Zehn Jahre Faschismus) et d'analyses linguistiques très personnalisées (ch. XXX : Der Fluch des Superlativs).

Klemperer se livre dans son journal, «Ich will Zeugnis ablegen». Tagebücher 1933-1945, à un double travail : ce spécialiste de littérature s'adonne sur le tas à la philologie ${ }^{1}$, tout en étant impliqué personnellement comme destinataire juif des messages d'exclusion et comme observateur des messages adressés aux Allemands non juifs.

Ma démarche sera double. Il s'agit, d'une part, de voir ce que représente la LTI pour Klemperer et de la replacer dans un mouvement allemand de la öffentliche Sprachkritik, 
telle qu'elle se manifeste dès « la césure » de $1945^{2}$, d'autre part, de montrer que, bien que Klemperer soit tributaire d'idées de son époque sur le langage que nous considérons aujourd'hui comme obsolètes, il a réussi à rassembler une documentation sur le langage de la période nazie, qui permet de voir à l'œuvre un jargon efficace.

Klemperer se montre conscient des changements linguistiques dûs aux changements historiques, sociaux et politiques d'une communauté linguistique et ressent le besoin de fixer un usage dont il pressent avec clairvoyance qu'il risque, un jour, d'être oublié. Il découvre que le langage de propagande est un ingrédient essentiel du fascisme. Dans une perspective très contemporaine, Klemperer porte témoignage, il dénonce le langage totalitaire comme un dévoiement de la communication par la pratique d'une parole à sens unique, sans possibilité de dialogue, qui permet tout juste la communication phatique, le dialogue feint avec la foule par la sujétion et l'applaudissement.

\section{Les termes Sprache/lingua/LTI}

5 La dimension affective de LTI, vocable personnel et crypté, montre qu'il s'agit dans ce relevé fait au jour le jour par Klemperer, d'une démarche de survie, d'une façon de continuer à vivre à travers l'écriture, en évitant tout sentimentalisme, toute tentative d'apitoiement sur soi-même. Le terme LTI Lingua tertii imperii finit par s'imposer (Zelle 89, 23.6. - 1.7.41, I 622), éliminant d'autres expressions épisodiques comme Sprache tertii imperii (30.6.33, I 35), Philologie der Nationalsozialisten (29.6.34. I 127), Syntax des 3. Reichs (17.6.36, I 287), Volkssprache des 3. Reichs (10. 1. 37, I 329).

6 LTI est employé à la fois comme langage chiffré, rappel parodique des nombreuses abréviations nazies (BDM, HJ...), visant à l'efficience technique, et surtout :

«als ein an mich selbst gerichteter sos-Ruf... (eine) Forderung an mich selber:

beobachte, studiere, präge dir ein, was geschieht - morgen sieht es schon anders

aus» (LTI, 13).

7 Le terme Sprache est ambigu, il peut désigner à la fois la langue comme système de signes et la parole (der Ausdruck) comme utilisation du système dans un acte individuel d'appropriation de la langue ${ }^{3}$. Ainsi le langage totalitaire ne représente qu'une variante propre à une époque d'utiliser l'allemand. Les Sprachkritiker d'après-guerre se sont donc trompés en assimilant une variété de l'allemand à la langue allemande. Il n'y a pas une langue nazie, qui pourrait être détachée de l'allemand, redevenu pur après 1945 ou définitivement entâché, mais des "Ausdrücke», des emplois du langage totalitaire tels que la période nazie en fournit un exemple particulièrement clair.

8 Nous autres contemporains, nous comprenons l'expression die Sprache des Nazismus comme die Sprache im Nazismus, c'est-à-dire un terme définissant l'emploi particulier de la langue allemande durant cette période du fascisme. Nous sommes maintenant convaincus "von der Unschuld der Sprache und der Schuld der Sprechenden $»^{4}(\mathrm{~K}$. Ehlich 1989, 27 et 1996). Pour nous, le langage n'est pas le reflet du monde, mais un système qui fonctionne seul, dans un rapport de simulation avec le monde, sans correspondance terme à terme, et qui informe aussi sur ceux qui le parlent en dévoilant leurs finalités. C'est pourquoi, H. Lübbe (Heringer, 1988, 48-70) ou P. von Polenz (Sternberger 1968, 246-269) sont fondés à critiquer la thèse centrale du «dictionnaire de la déshumanisation »: "Der Verderb der Sprache ist der Verderb des Menschen», 
car une critique langagière qui s'exerce sur des mots n'est pas une critique du langage politique, mais une critique politique et morale de la réalité qu'ils désignent.

Klemperer partage la conception des Sprachkritiker traditionnels sur le langage; à

l'instar d'un Karl Kraus, il est persuadé que le langage a vocation à révéler le vrai :

«LTI. Die Sprache bringt es an den Tag. Bisweilen will jemand durch Sprechen die

Wahrheit verbergen. Aber die Sprache ist wahrer als er. » (31.3.42, II 58).

Il se révèle tributaire de son époque partant d'une conception (die Abbildtheorie) qui veut qu'il y ait adéquation entre les mots et les choses, en confondant la langue et ceux qui la parlent. Mais sa position sur ce point est plus claire que celle des Sprachkritiker d'après-guerre. En forgeant ce terme personnel de LTI, ce n'est pas la langue allemande qu'il condamne, mais une de ses variétés totalitaires. LTI permet le mensonge, la manipulation et la diffamation :

« Das Gift ist überall. Im Trinkwasser der LTI wird es verschleppt, niemand bleibt

davon verschont. » (LTI, 101)

11 Certes, mais le langage fournit aussi toutes les ressources pour dévoiler la manipulation et pour résister à l'usage ambiant.

Rétrospectivement, la documentation linguistique rassemblée par Klemperer dans son journal intime daté de 1933 à 1945 constitue un jalon de la Sprachkritik, discipline propre à l'Allemagne. La définition actuelle de cette discipline :

«die Sprachkritik (wird) gesehen als Untersuchung eines sprachlichen

Zeichensystems auf seine kommunikative Effizienz » (Heringer 1988),

13 s'applique bien au témoignage direct (Klemperer) et pas du tout à l'étude rétrospective du Dictionnaire de la déshumanisation, car ces Sprachkritiker d'après 1945 sont plus sensibles aux expressions qu'au système dans lequel elles s'insèrent. Il est d'ailleurs surprenant de constater que peu de termes critiqués sont communs ${ }^{5}$ : En effet les Sprachkritiker d'après 1945 relèvent en majorité des termes abstraits du langage moderne caractéristiques d'une société évoluée et technocratique comme Ausrichtung, Gestaltung, Betreuung, Vertreter, Einsatz..., à côté de quelques termes idéologiquement plus marqués comme : charakterlich, Härte, Lager, alors qu'on trouve, à l'inverse, chez Klemperer une abondance de termes marqués par l'idéologie nazie comme fanatisch abondamment employé dans Mein Kampf et dans les Tagebücher de Goebbels -, Ahnenpaß , AD (Arbeitsdienst) Ariernachweis, BDM, Gestapo, Jud, Kraft durch Freude, Konzentrationslager, Machtergreifung, SA, SS, Systemzeit, Untermensch, Winterhilfswerk, etc, autant d'expressions qui font leur apparition dans les Duden ${ }^{6} 11$ (édition de 1934) et 12 (1941), donc des termes qui sont tributaires d'un jargon idéologique, et seulement quelques éléments qui montrent l'évolution de l'allemand vers un langage plus technochratique et qui évolue sous l'influence des médias.

Klemperer s'explique à plusieurs reprises sur le pouvoir du langage idéologique et sur le changement linguistique. À la manière des Sprachkritiker d'aujourd'hui, il tente d'interroger le langage sur sa capacité à dire la réalité de ceux qui l'utilisent en montrant que le même lexème peut apporter des informations différentes, étrangères au référent, portant sur le locuteur et sa situation de communication; le terme, dans ce deuxième cas, peut bien avoir préexisté au nazisme, il n'en connote pas moins, après coup, l'appartenance à ce système de pensée du fait de son utilisation idéologique. À propos du vocable Untermensch, rencontré dans der Stechlin de Fontane, il constate ainsi : 
« Neue Wörter. Sie sind neu in dem Augenblick, wo sie als Ausdruck einer neuen Gesinnung oder neuen Sache auftauchen und in Mode kommen. Insofern ist 'der Untermensch' doch ein spezifisches und neues Wort in der Sprache des dritten Reiches. » (26.12.40, I 567).

À côté de quelques néologismes, qui concernent essentiellement des institutions nouvelles, le vocabulaire incriminé relève, la plupart du temps, du phénomène du changement sémantique.

\section{Les différentes strates du langage}

La méthode utilisée par Klemperer est empirique : il relève des termes «Einzelworte, Redewendungen, Satzformen» (LTI, 21), des Ausdrücke qui le frappent au quotidien. Comme critères de sélection privilégié, il indique la fréquence et le changement linguistique (deux critères combinés pour fanatisch - terme auquel il consacre un article dans LTI - mais aussi la charge sémantique.

Je vais tenter de regrouper des termes relevés et de les décrire selon les catégories de la linguistique de l'énonciation, de la pragmatique et de la Sprachkritik actuelle dans la perspective énoncée par W. Dieckmann ${ }^{7}(1969,81)$ à propos du langage politique.

\section{1. Le lexique idéologique (Meinungssprache)}

Il s'agit, d'après J. Klein $(1989,7)$ de ces mots du champ politique et social :

« in denen, politische Gruppierungen ihre Deutungen und Bewertungen der politisch-sozialen Welt, ihre Prinzipien und Prioritäten formulieren. »

Certains termes sont des créations de l'idiolecte nazi, des néologismes de forme, car ils désignent des institutions nouvelles: SS, SA, national-sozialistisch, Gauleiter... et des modes de pensée propres : ainsi en va-t-il des créations de vocabulaire concernant la Rassenlehre (aufnorden, artfremd... cf. LTI, 200). Mais, dans la plupart des cas, des termes plus anciens réapparaissent en changeant de sens (cf. Konzentrationslager LTI, 42).

LTI lie certains termes avec d'autres pour créer des stéréotypes, cf. « die Überwindung des 'faden Intellektualismus' "(17.4.35, I 192) qui sont constamment répétés, en surinvestissant certains vocables positivement (Volk, Blut, Raum, Art, Rasse etc) et d'autres négativement (Jude). Klemperer relève donc un type de mots [Rasse/ «rassisch » (14.10.40, I 557/ 4.7.42, I 109), Blut, Art (12.6.42, II 124)] spécifiquement nazis, « nicht nach seiner Formung, aber seiner neuen Anwendung nach.» (LTI, 47). Le changement linguistique (Sprachwandel) produit donc surtout des Neuprägungen. Ce sont des néologismes de sens, un signifiant ancien étant doté d'une acception nouvelle. Un des tenants du régime, Norbert Pechau, qui a écrit en 1935 une thèse intitulée: Nationalsozialismus und deutsche Sprache, se montre, d'ailleurs, conscient de cette caractéristique :

«Das Hauptgewicht der nationalsozialistischen Sprachbeeinflussung liegt auf der neuen Sinngebung oft alter bekannter Wörter $»^{8}$.

\section{a) Les connotations axiologiques}

21 Certaines unités linguistiques fournissent un jugement d'appréciation ou de dépréciation porté sur l'objet dénoté, ainsi sonnig dans les faire-parts de décès : « unser 
sonniger edler Sohn, der sonnige Vater unserers Töchterchens»(26.7.41, I 654), kämpferisch, Aufbruch, (6.8.41, I 657), blond, sont-ils marqués positivement :

«Christus, der blonde und blauäugige - blond ist Lieblingswort, einmal das blonde

Blut», $(13.6 .42,122)$

alors que polnisch (« eine polnische Organisation » 20.9.39, I 490) ou Systemzeit désignant la période de Weimar (12.7.38, I 415) sont dépréciatifs.

Peut-on qualifier un langage idéologique de "connotatif $»^{9}$ ? Dans la mesure où, dans cet usage, le signifiant est moins le terme choisi que le fait de l'avoir choisi, la réponse est affirmative. À cet égard, les termes les plus frappants sont ceux qui changent de valeur axiologique comme hart, stur, il en va de même de blindlings :

(es) «gehört zu den Pfeilerworten der LTI, es bezeichnet den Idealzustand nazistischer Geistigkeit ihrem Führer und ihrem jeweiligen Unterführer gegenüber » (LTI, 162).

« Man muß dem Führer blindlings die Gefolgschaft leisten, blindlings!» - (24.11.36, 322).

Klemperer s'attache au néologisme de sens à propos de fanatisch; ce terme, utilisé avant et après le nazisme au sens dépréciatif de «frénésie sectaire » est employé de manière purement laudative, au sens de leidenschaftlich pendant le national-socialisme, comme l'indique la note suivante :

«Sprache tertii imperii : Neujahrsbotschaft Lutzes an die SA... - Zweimal : unser 'fanatischer Wille' im nicht-pejorativen Sinn. Betonung der Gläubigkeit ohne zu verstehen. (1. 'fanatischer Einsatz der SA', 2. 'fanatische Einsatzbereitschaft.' » 1.1.39, I 174).

Des termes du langage commun fonctionnent sur le mode de la connotation positive ou négative par collocation, par association avec des termes valorisés : «der fanatische Glaube an den Endsieg» (LTI 65), " 'fanatisch deutsch' eine contradictio in adjecto, 'fanatisch' ein Lieblingswort Hitlers » (17.8.42. II 209) ou des termes dévalorisés : « die internationale Juden-Kanaille » (17.4.35, I 192).

Toute infraction à cette loi d'homogénéité fait l'effet d'un dérapage, comme celui-ci : « in einer Lokalbetrachtung des 'Freiheitskämpfers' gab es neulich eine Entgleisung oder einen Rückfall ; es war von den 'fanatisiserten russischen Horden' die Rede, wo dann das geheiligte 'fanatisch' wieder ins Unheilige zurückgesunken war. » (11.2.43, II 327). Le néologisme de sens ne se maintient donc pas toujours durant la période national-socialiste. La question $\mathrm{du}$ changement linguistique et du signifié de connotation est donc au centre de l'analyse. Ainsi Klemperer décèle-t-il un changement linguistique dans l'emploi du terme : charakterlich, placé au centre d'une constellation manifestant l'idéologie nazie, "charakterlich gut " (LTI, 206) signifiant: einwandfrei nazistisch. En soi un mot neutre, charakterlich, est utilisé de manière purement idéologique :

« Zur Erklärung des LTI-Wortes: Charakter ('Gesinnung') ist so wesentlich, wesentlicher als Geist und Wissen, daß man ein neues Adjektiv bildet, um Menschen $\mathrm{zu}$ werten. In den Schulprüfungen und -zeugnissen wird vor allem das 'Charakterliche', d.h. die nationalsozialistische Prägung des Schülers bewertet. » (24.1.43, II 319-320).

Un autre exemple de changement de connotation axiologique est l'emploi mélioratif du qualificatif stur, employé généralement de manière dépréciative, mais exclusivement de manière laudative par Hitler et consorts (8.1.39, I 455/ 6.3.39, I 464/18.10.39, I 498). 
L'inversion des valeurs connotatives est donc une composante du langage nazi notée par Klemperer.

La pratique connotative axiologique s'attache en particulier à la formation des mots formés à partir d'un même radical qui fonctionnent comme antonymes. S'opposent ainsi des mots préfixés, suffixés ou encore des mots composés :

\begin{tabular}{|l|l|}
\hline Emploi laudatif ou neutre vs & péjoratif \\
\hline liberal & /liberalistisch (modèle: marxistisch?) \\
\hline intellektuell & /intellektualistisch \\
\hline deutsch & /entdeutschen \\
\hline Arier & /Nichtarier \\
\hline deutschstämmig & /Judenstämmling \\
\hline Volksgemeinschaft & /-schädling \\
\hline Volkskanzler & /-verrat \\
\hline volksnahe/-bewußt & /-fremd etc. \\
\hline
\end{tabular}

Un type de préfixe, qualifié par Klemperer de distanzierende Vorsilbe (LTI, 7) apparaît très productif, il s'agit de :

- ent- machten (9.12.39, I 503)/juden, (9.10.37, I 382), -artet (« entartete Kunst» 16.3.42, II, 43), - ver-judet/niggert (23.6.40, I 534)/jüdeln (4.3.41, I 582).

familles de mots connotées positivement (1) ou négativement (2) donnent lieu à une inflation de mots motivés formés à partir de certains radicaux connotés :

(1) Held, heldenhaft, heldenmütig, (« unsere heldenhaft kämpfenden Truppen » LTI, 14),

(2) Weltmacht, Wehrmacht Defensives «Reichswehr» zu aggressivem (sic.) Wehrmacht. Macht ein Lieblings- und Stammwort wie Volk. 8.7.41, I 645)

La famille de mots la plus riche en désignations, et c'est bien significatif, concerne le groupe stigmatisé par les nazis comme l'adversaire par excellence, les juifs :

(2) Jude, jüdisch, jüdische Menschen, entjuden, judenfrei, Voll-/Halbjude, Judenstämmling, jüdisch-marxistisch, jüdisch-bolchevistisch, jüdisch-kapitalistisch, jüdisch-englisch, jüdischamerikanisch...

Les connotations les plus négatives s'attachent aux Schimpfwörter: Jud ${ }^{10}$, Juda, Alljuda, Judenknechte, Juden-Kanaille, Juden-Sau...

On est témoin d'une polarisation du vocabulaire qui atteint même les mots du vocabulaire commun. Klemperer, bien qu'il soit sensible à ce phénomène, ne peut se préserver totalement de la polarisation du vocabulaire, destinée à créer des stéréotypes positifs ou négatifs, assurant : «Deutsch sein heißt Tier sein » (11.9.34, I 143), parodiant ainsi le slogan nazi : «Deutsch sein heißt klar sein ». La polarisation incessante, la 
dichotomie du bien et $\mathrm{du}$ mal, permet aux propagandistes nazis, en disant le "notoire », de ne plus avoir à prouver ce qu'ils désignent par un vocable marqué, donnant ainsi à entendre qu'ils sont du côté du positif, l'adversaire (les juifs, les bolcheviques...), du côté du négatif.

\section{b) Les euphémismes (Schleierworte)} pour holocauste).

Alors que la métaphore dit ce qui ne peut avoir de synonyme ou de paraphrase, mais représente le discours le plus pertinent en situation, les euphémismes sont des termes qui ne changent pas le dénoté mais en masquent la réalité (par ex. Sonderbehandlung

Il s'agit, parmi les termes relevés dans le journal, essentiellement de désignations destinées à cacher aux Allemands le sort réservé aux juifs. Elles sont utilisées en les débarassant de toute charge émotionnelle désagréable pour tenter, par l'intermédiaire des images associées, d'influer sur le dénoté. Ainsi, la convocation à la Gestapo est-elle euphémisée, ce que Klemperer signale par l'emploi de guillemets et de commentaires :

«Kreidl sen. vorgestern morgen 'zur Befragung' auf die Gestapo bestellt, nicht zurückgekommen. » (21.11.41, I 687).

Il évoque « die Tortur des 'Bestelltseins’ » (26.4.1943, II 361) :

«'Hinbestellen' - auf stundenlange Spaziergänge schicken, sich immer wieder zu neuen Beschimpfungen und Püffen melden lassen ist die übliche Tortur im Anschluß an die Hausdurchsuchung ». (8.5.42, II 79)

Ainsi qualifie-t-on d'Evakuierungen les déportations vers l'est, de größte Industriestadt des Ostens le ghetto de Litzmannstadt (7.2.41, I 693). Klemperer emploie le terme de NSJudendeutsch, une variété de LTI, pour évoquer des euphémismes employé par les juifs comme verreist sein pour sitzen, Konzertlager pour Konzentrationslager ou KZ (1.1.42, II 5), un euphémisme obligé, en relation avec sa situation personnelle de juif marié à une aryenne est celui de begünstigt (sein) :

«In der Liste der letzten fünfundvierzig 'Alten'... der Studienrat und Leichenbestatter. Er ist in meinem Alter, aber als Kriegsbeschädigter 'begünstigt'.

Den andern ist Zwangsarbeit in Polen zugedacht. » (16.9.42, II 245)

Ainsi, l'envoi à Theresienstadt qualifié de (Reichsaltersheim) est présenté comme un "privilège", mais la plupart seront déportés à Auschwitz en 1944. Cependant, l'agravation de la situation rend le vocabulaire plus transparent quand on passe de l'euphémisme "Juden unerwünscht» (5.2.39, I 461) du début des lois raciales au «Juden verboten » $(10.8 .41$, I 659$)$ du temps de guerre.

Évoquant les déportations massives, Klemperer rétablit le véritable dénoté en jouant sur l'euphémisme abgewandert utilisé par exemple pour masquer la déportation de Caroli Stern Hirschberg :

«Beachte zu LTI 'abgewandert' für abgewandert worden. Harmloses Wort für

'vergewaltigen', 'vertreiben', 'in den Tod schicken'. » (27.2.43, II 335).

Les euphémismes sont donc une forme cynique de mensonge. Ce terme de Lüge revient d'ailleurs tout au long du journal. Il s'applique particulièrement bien quand Hitler justifie toute mesure arbitraire et cruelle en se référant à «das gesunde Rechtsempfinden des Volkes» (6.12.38, I 441), le mensonge est utilisé autant pour conquérir le pouvoir : 
«Als Hitler seine Feinde 'standesrechtlich' erledigt hatte, ließ er durch seinen Reichsrat beschließen, alles Getane sei 'rechtens' geschehen. Die knappste, affirmativste, germanischste Rechtsformel als Gegengift. » (4.11.34, I 161),

que, par la suite, quand il s'agit d'«informer » sur la situation :

«Sie behaupten mit kalter Stirm im Abendblatt das Gegenteil dessen, was sie im Morgenblatt behauptet haben, und das Volk frißt alles. Sie haben Röhm und Heß und Stalingrad überstanden. » (1.1.44, I 467)

\section{c) Les mots dans l'air du temps (Modewörter)}

Les mots « actuels ", à la mode, véritables mots-témoins, éléments à la fois expressifs et tangibles qui concrétisent un fait de civilisation, ces «Pfeilerworte der LTI», dans lesquels le nationalsocialisme reconnaît son idéal, sont des termes du langage commun qui fonctionnent comme des expressions stéréotypées par la polarisation généralisée.

Klemperer constate ainsi le surinvestissement de "gleichgeschaltet ${ }^{11}$, terme valorisé par le nationalsocialisme, " ein heiliges Wort, ein Symbol », (19.8.33, I 49). Sont ainsi polarisés et investis positivement: weltanschaulich//Weltanschuung (LTI, ch. XII), Aufbruch (einer Nation) (T 1. 132), Bewegung « München, Hauptstadt der Bewegung ». (10.5.38, I 407), Aktion (7.8.44, II 557-558), völkisch (LTI, 205), mot le plus spécifique du nazisme, selon J. P. Faye.

Le surinvestissement de l'événement, qui donne lieu à de l'émotion, qui est porteur d'affect, met à la mode des mots comme erleben/Erlebnis pour mettre en relief l'idée que le peuple allemand vit une ère exceptionnelle. Ainsi Die Reichstheaterwoche in Dresden reçoit-elle l'attribut magnifiant de : «das Erlebnis von Dresden » (13.6.34, I 115). L'idée de la participation permanente de tout l'être aux moindres actes de la vie sociale présentés comme une fête fait partie d'une stratégie d'élévation de la réalité quotidienne qui agit comme un narcotique :

«Die LTI zieht die Dinge geflissentlich in die Späre des Erlebens'. Jugend erlebt Wilhelm Tell' » (LTI, 258).

\section{d) Les mots savants}

C'est, selon Klemperer, pour en imposer au peuple par des mots qui ne sont pas compris par lui que les dignitaires du régime, le Dr. Goebbels et leurs acolytes, parsèment leurs discours de mots étrangers, entraînant, d'ailleurs, les critiques des puristes du Deutscher Sprachverein, organisme soucieux d'éliminer de l'allemand les mots étrangers et qui critique les termes comme Propaganda (Werbe), Konzentrationslager (Zwangslager), Sterilisation (Unfruchtbarkeitmachung).

51 Certains mots étrangers sont polarisés, trahissant la position qu'occupe l'énonciateur nazi ; sont ainsi valorisées les lois de Nuremberg qualifiées de « säkulär »:

«Die Judengesetze hat Hitler in Nürnberg 'säkulär' genannt (Fremdwort und

Unbildung und Größenwahn Thema des 3. Reiches. Cf. Garant.) » (5.10.35, I 222)

Garant sonne mieux que Bürge "magisch wie Kirchenlatein » (11.5.40, I 524). Les termes de Terror (Luftterror, Bombenterror) et d'Invasion (LTI, 268) sont bien connus; sont désignés du doigt die Aggressoren Frankreich/England (11.5.40, I 524) :

«Frankreich... während der Offensive, in immer steigendem Maß 'verjudet',

'verniggert', dekadent', sadistisch" » (23.7.40, I 534). 
Die jüdische Plutokratie $(\mathrm{T} 1,485)$ paraît d'autant plus redoutable qu'on utilise un terme totalement incompréhensible.

L'époque témoigne d'une inflation de verbes en -ieren : diskriminieren, kastrieren : "Der Bolschevismus kastriert das Individuum » (1.5.43, II 366), diffamieren, riskieren, sabotieren, autant de comportements attribués aux adversaires et en particulier à la « parasitäre Rasse » (LTI 191).

Un second motif d'emploi des mots étrangers tient au camouflage sur les buts poursuivis. Le linguiste, P. von Polenz (1967, 119-120), met en lumière : «(die) Suggestivkraft und Tarnwirkung bewußt gewählter Fremdwörter der politischen Propaganda. »Cette seconde fonction est parfaitement décelée par Klemperer :

« Was Hitler furchtbar genau kennt und in Rechnung stellt, ist stets die Psyche der nichtdenkenden und in Denkunfähigkeit zu haltenden Masse. Das Fremdwort imponiert, es imponiert umso mehr, je weniger es verstanden wird; in seinem Nichtbegriffenwerden beirrt und betäubt es, übertönt eben das Denken. » (LTI, 268) «Beachte die jetzt immer häufigere 'Neuordnung Europas', nicht mehr die Freiheit Deutschlands, sondern die euphemistisch umschriebene europäische Suprematie. » (10.4.1941, I 586)

En ce domaine, une certaine systématisation apparaît parfois, quand on germanise Sanitäter en Feldscherer (29, 8.39, I 479), sans doute au profit des connotations guerrières. Mais, mis à part quelques germanisations curieuses notées dans LTI, 266 : Bestallung (Approbation), Entpflichtung (Emeritierung), Belange (Interessen), une germanisation systématique est évitée car elle nuirait aux objectifs de la propagande et Hitler interdira par décret, le 19 novembre 1940, toute lutte contre les mots étrangers et toute germanisation forcée ${ }^{12}$.

7 En redistribuant les termes relevés par Klemperer selon différentes rubriques, on ne peut échapper à des classifications croisées, ainsi le terme de «europäische Suprematie » est à la fois choisi parce qu'il est savant, mais il représente aussi un déguisement pudique, donc un euphémisme pour une germanisation de l'Europe.

\section{e) Les métaphores}

L'idéologie ne se contente pas d'influer sur l'aspect connotatif du langage, elle investit à travers la métaphore jusqu'au langage dénotatif. la métaphore est l'expression qui crée le plus d'effets dans un certain contexte. Elle permet d'évoquer un univers conceptuel en cristallisant sur une image médiatrice des valeurs affectives. C'est donc un phénomène à la fois dénotatif et connotatif.

9 Dans l'optique d'un conditionnement qui en dit long sur la véritable nature du régime, les métaphores guerrières véhiculées par des substantifs comme: Käuferschlacht, Erzeugerschlacht (13.12.36, I 325-326), Arbeitskampf, Regenfront (22.1.39, I, 460), Strafexpedition (LTI, 49), ou par des verbes « der deutsche Spielfilm marschiert » (14.9.36, I 305) sont remarquables.

60 Il opèrent en effet sur le dénoté lorsque les nazis forgent des métaphores comme : rote «Horde »/bolchevistische/russische «Horden» (11.2.43, II 327) pour désigner l'adversaire russe, parlent de «Banden » pour qualifier les résistants qu'il faut « liquidieren » (4.8.44, II, 556) ou encore utilisent les termes propres aux animaux en parlant aux juifs : « Dein Wurf ist uns entkommen, wir wollen dich fertigmachen» (LTI, 5), il s'agit d'éliminer, 
d'éradiquer (ausrotten) les juifs, les communistes (14.7.34, I122) « (sind) wie Flöhe und Wanzen (zu) vertilgen » (21.7.35, I 209).

61 Comme le remarque Heringer $(1988,19)$ :

«Die Rede von Juden als Parasiten ist ein gutes Beispiel, wie Metaphern Realität konstituieren können, ihre eigenen Kreativität und Dynamik in sich tragen. »

Le conditionnement par l'usage répété de la métaphore, qui personnifie des abstractions et animalise les adversaires (Animalisierung des Menschen LTI, 150), fait apparaître les juifs sous l'espèce biologique de transmetteurs de maladie qui attaqueraient le Volkskörper sain, ce qui justifie tout un ensemble de métaphores qui se cristallisent autour de la maladie et des moyens de guérir en s'en débarrassant (Judenvertilgung). Les juifs sont qualifiés de Parasiten, de Volksschädling:

« Darum besinnt Euch auf Eure Pflicht gegenüber der Volksgemeinschaft, auf daß der Vorwuf der Volksfremdheit für Euch nicht zur Beschuldigung als Volksschädling werde ! » (16.12.34, I 169),

pour éliminer die parasitäre Rasse (LTI 191), il y a la gestapo qualifiée de Volksschädlingsbekämpfer :

"Scherner berichtet gestern: Die eigentliche Bezirksgestapo residiere in Plauen; hier hielten sich... sechzig 'Volksschädlingssbekämpfer' auf, vermutlich eine Gruppe von Beobachtern, Spionen, Spitzeln, Polizeihunden, nach rebellischen Flüchtlingen und Deserteuren stöbernd. » (10.3.1945, II 699).

Klemperer relève dans un discours de Goebbels ce mot programmatique :

Wir haben die Juden «ausgemerzt », (2.12.37, I 385), idée qui réapparaît sous différentes formes comme beseitigen (23.11.44, II 615), ausrotten :

«Der Verwalter unseres Hauses, erzählte Frau Ziegler, sei wegen Judenfreundlichkeit vor die Gestapo geladen worden. Auf seine Erklärung, die Leute seien anständig, wurde ihm gesagt, es gebe keine anständigen Juden und die' ganze Rasse werde ausgerottet werden' ». (29.8.42, II 224)

L'objet métaphorisé, déshumanisé devient porteur d'une nouvelle propriété qui le définit. Il est encore situé plus bas dans l'échelle des êtres qu'un Untermensch.

Les métaphores de la déshumanisation sont légion dans le journal ou LTI, cf. RathenauBeseitiger (T1, 43), das Amtswort Kadaververwertung (LTI 159). Ce type de métaphores qui animalisent l'humain sous des espèces particulièrement répugnantes et nuisibles, à éliminer avant que la contagion ne gagne, ont donc une fonction centrale dans la construction idéologique qui cristallise dans «le parasite» une façon d'envisager le réel. La métaphore a donc une manière particulière de signifier l'objet. Ce point de cristallation dans le mot frappe Klemperer :

«Denn unter einem Einzelwort erschließt sich dem Blick das Denken einer Epoche, das Allgemeindenken, worein der Gedanke des Individuums eingebettet, wovon er beeinflußt, vielleicht geleitet ist. » (LTI, 158).

On peut toujours se soustraire au langage idéologique connotatif en évitant d'y avoir recours, il est plus difficile d'échapper à l'entraînement métaphorique, ainsi à propos de l'arrestation du maire de sa ville, Klemperer remarque-t-il :

"Alle kleinen Leute gebrauchen immer denselben Ausdruck: er ist 'verseucht' ». $(20.6 .37$, I 361)

Il est très conscient de cette biologisation généralisée de langage :

« 'völkisch' im Sinne der Reinblütigkeit ist ein zoologischer Begriff und ein Begriff, dem längst keine Realität entspricht, jedenfalls noch weniger Realität als der alten 
strikten Unterscheidung zwischen den Sphären des Mannes und Weibes. » (10.1.39, 457), relever des idées dominantes de l'époque. Ce vocabulaire se caractérise par la tendance à l'abstraction. Il s'agit de termes voisins de ceux qui seront aussi critiqués par les Sprachkritiker d'après-guerre qui dénoncent leur «caractère inhumain ». Klemperer stigmatise ce qu'il nomme eine Entindividualisierung (17.1.42, II 27), qui lui paraît significative d'un langage de masse prôné par les nazis. Il s'applique aussi bien à des termes comme Ausrichtung, Einsatz (8.1.39, I 455), « Arbeitseinsatz » (17.1.42, II 11), qu'à Einstellung (16.12.39, I 505), ou Betrieb (9.8.41, I 645), Betreuung (LTI, 252), Nachwuchs (16.7.38, I 411), aufziehen « etwas groß aufziehen » (23.6.-1.7.41, I 631), ankurbeln (22.3.42, II 51), organisieren/ Organisation (LTI, 106), verankern (LTI, 162), dont l'emploi surabondant lui paraitt témoigner d'une intentionalité cachée :

«Jeder soll Automat in der Hand des Vorgesetzten und des Führers werden » (LTI, 162).

Tout d'abord, il convient de faire la différence entre ces termes lorsqu'ils sont employés en liaison avec des termes connotés, à la manière du langage idéologique et deviennent alors valorisés ou dévalorisés: "groß aufgezogene Aktion» (LTI, 53), « die jüdische Intelligenz » (LTI, 113), et lorsqu'ils apparaissent comme les témoins d'un langage technocratique émergeant au cours du $\mathrm{xx}^{\mathrm{e}}$ siècle (cf. die Wirtschaft ankurbeln). Il y a une tendance, dans l'allemand de la deuxième partie du siècle, à nominaliser les verbes 
d'action, à utiliser un grand nombre de substantifs déverbatifs comme Aufbau, Aufstieg, Vorstellung, Berufsfindung..., évolution qu'H. Eggers ${ }^{15}$ commente ainsi :

«Abermals bestätigt sich [...] die moderne Neigung, den Aussageinhalt in die substantivischen Satzglieder zu verlegen.»

Le style nominal, que l'on reconnait bien à l'oeuvre dans des expressions connotées comme « die national-sozialistische Ausrichtung der Wissenschaft » (11.2.43, II 327), va de pair avec un effort de concentration dans l'information, ce dont l'inflation des sigles (BDM, HJ), parodiée dans LTI, fournit un autre exemple.

Klemperer, qui ne peut pas, à son époque, appréhender la distinction entre Meinungssprache et Funktionssprache, forge des caractéristiques ad hoc parlant d'« américanisation » du langage, de « mécanisation, matérialisme, désindividualisation »... Sur ce point précis, Klemperer fait montre de certains travers de la Sprachkritik d'aprèsguerre rappelés par P. Braun ${ }^{16}$ qui, par ailleurs, reconnaît leurs mérites :

"Sie thematisieren im weitesten Sinne den Zusammenhang von Sprache und Gesellschaft.... Die Sprachkritik versteht Sprache als gesellschaftliches Ereignis, aber sie behandelt die in diesem Zusammenhang aktuellen Probleme oftmals vorwissenschaftlich und intuitiv.»

Mais on ne peut guère leur en faire grief à une époque où la linguistique ne considère pas l'analyse du discours comme faisant partie de son champ d'investigation.

Klemperer ne se contente pas de relever des vocables qui heurtent sa sensibilité au quotidien. Il donne les moyens de comprendre, à travers cette documentation, certaines techniques du langage totalitaire.

\section{Aspects rhétoriques et stylistiques de la propagande (nazistische Stilistik)}

\section{a) L'amplification}

Elle consiste à augmenter l'expression en lui donnant l'aura de l'exceptionnel ${ }^{17}$ : Klemperer utilise pour évoquer cet effet les termes péjoratifs de Schwulst, Bombast, Barnumiade, qui lui paraît caractériser ce qu'il dénomme den "Größenwahn » (5.10.35, I 222) du nazisme, « der Wesenskern der LTI» (12.7.44, II 545). En voici un bel exemple qui combine la métaphore filée et l'amplification :

« Der Spruch selber, von Goebbels, lautet : 'Nur ein eisernes Geschlecht wird sich im Sturme unerer Zeit behaupten können. Es muß Eingeweide aus Eisen und ein Herz aus Stahl besitzen. »19.7.44, 547)

81 Plusieurs moyens linguistiques sont mis à contribution pour obtenir cet effet, comme la gradation par :

82 - la comparaison, la superlativation et l'emploi de l'élatif (« der Fluch des Superlativs » LTI, 228-238) : « die abscheulichste Rasse » (7.4.39, I 467), « der schlimmste Winter seit hundertvierzig Jahren (28.4.42, II 74), « die größte Autobahn der Welt » (8.8.37. I 372), « das größte Bombardement der Weltgeschichte (über London)» (21.10.40, I 558), « das Jahr 1941 wird die Vollendung des größten Sieges unserer Geschichte bringen» (31.12.40, I 568), « Bialystok-Minsk, 'die größte Umfassungs- und Materialschlacht der Weltgeschichte' » (21.10.40, I 648), « die tiefsten Quellen deutschen Wesens » $(\mathrm{T} 2,542)$. 
83 Certains exemples sont particulièrement tautologiques et emphatiques comme : " 'das größte Bombardement der Weltgeschichte', vor zwei Tagen war 'Bartholomäusnacht für London'. » $(1.10 .40,558)$,

84 - l'emploi de chiffres énormes: «Das Tausendjährige Reich» (LTI, 232), « Hundertfünfzigprozentig » (12.7.41, I 647), « Der Oberbürgermeister Goerderler... seit 20. Juli flüchtig... eine Million Belohnung » (4.8.44, II 556),

85 - l'utilisation d'épithètes à valeur absolue : ewig "wir haben den Weg in die Ewigkeit gefunden» (18.1.38, I 393), triumphal, tief " ein triumphaler Empfang, die tiefe Beglücktheit, die größte Dankbarkeit » (10.5.38, I 406), einmalig "Görings einmalige Verdienste » (20.10.40, I 566), total, un des mots-clé qui exprime de manière synthétique les idées du nazisme : "die totale Erziehung, die totale Abschnürung der Juden, das totale Spiel... » (3.5. 44, II 511), « der totale Krieg » (LTI, 123),

86 - l'utilisation de termes à connotation religieuse comme heilig et du langage de la foi religieuse : »Heute die Wahl, 'der Tag des großdeutschen Reiches'... - die heilige Wahl » (référendum sur l'annexion de l'Autriche. 10.4.38, I 401, 402), « der heilige Krieg » (LTI, 117),

87 - l'emploi de déterminants ou de déterminés de mots composés augmentatifs ou énonçant une valeur absolue: "Großdeutschland», "die Großkundgebung, die Großkantine » (12.6.44, II 545), "die Vernichtungsschlacht » (LTI, 234), de suffixes : «die pausenlosen Vergeltungsangriffe » $(21.10$; 40, I 558), «'zahllose Divisionen' zu neuen Operationen' aus der Abwehrfront' » (6.6.40, I 533). Cependant l'accumulation de termes surévalués, ainsi survalorisés, tend à en émousser complètement l'effet et même à provoquer l'effet inverse.

\section{b) La pseudoargumentation}

Elle repose souvent sur le mécanisme de la redondance sémantique et sur la tautologie (ex. : « für alle ewigen Zeiten »). Klemperer en relève les effets à propos du mythe du sang et de la valorisation de la rasse :

Beachte im Mythus ${ }^{18}$ « eine rassisch gebundene Art = unterbewußte Denkungsart. Art ist vorhanden in Denkungsart, ebenso als Tierart - aber in der Überbetonung wie hier ist es neu. Es ist so überbetont als Rasse, daß Verfasser gar nicht die Wiederholung des gleichen Wortes spürt. » $(4.5 .40$, I 110)

89 Il s'agit simplement de marteler les mêmes termes en allant jusqu'à la répétition du même dans la tautologie pour en assurer l'effet de persuasion.

90 L'argumentation cachée se loge dans les slogans ${ }^{19}$ et les locutions. Le slogan est une forme spécifique à la propagande politique qui s'intéresse à la réception, pour attirer l'attention, avoir un effet mémoriel et persuasif sur l'allocuté. Les slogans politiques expriment, sous forme condensée, des attitudes et opinions idéologiques dont le martèlement doit servir à influer sur les récepteurs. C'est un instrument du combat idéologique qui cherche à favoriser la polarisation de la vie politique et sociale et à empêcher la réflexion critique.

91 Les dénominations programmatiques comme der Nationalsozialismus, das dritte Reich... fonctionnent comme des slogans. Klemperer relève comme tels des formules comme Tausendjähriges Reich, die Friedensrede (Hitlers) (9.7, 33, I 39), der Volkskanzler, die Befreiungstat Hitlers (6.3.36, I 250) - terme désignant l'occupation de la rive gauche du Rhin. Ainsi der Blitzkrieg est-il qualifié de «neuestes Schlagwort»: «In sechs Wochen 
ist England vollkommen erledigt ! » (8.5.40, I, 522), mais Klemperer note aussi der totale Krieg (20.4.43 II, 345, der Endsieg (2.8.44 II, 555).

Les slogans contiennent implicitement une structure argumentative : c'est parce que nous sommes les forts et les mieux préparés par notre gouvernement que nous allons mener une "guerre-éclair", nous menons une guerre défensive (Gegenschlag), ... argumentations inattaquables de ne pas être formulées. Cette argumentation implicite convient donc particulièrement aux arguments faibles.

Parfois l'argumentation apparaît clairement dans des affirmations programmatiques comme « Hitler ist Deutschland» (11.9.34, I 143), " Mit unseren Fahnen ist der Sieg » (LTI, 93). La répétition du même ne semble même pas être perçue :

«Wir sind unseren Feinden gegenüber auf Grund unserer besseren Volksrasse sehr im Vorteil.' » (11.7.44, II 543)

Les locutions et rituels : «Blut und Boden » (9.10.34, I 154), « mit deutschem Gruß», « Heil Hitler » etc, servent, de même, à construire ou à conforter des stéréotypes.

\section{c) Le caractère appellatif}

Dans le discours politique transmis dans les journaux et à la radio, Klemperer note le lien direct instauré entre le Führer (der Volkskanzler) et la Volksgemeinschaft par l'adresse et l'emploi d'indices d'allocution tels les vocatifs, les appellatifs, les pronoms personnels de première et deuxième personnes : ce tu offert aux Volksgenossen :

« Du gibst dem Führer dein Ja. » (21.11.34, I 165). «Dein Handeln und Charakter (sic) bestimmt das Blut... / Hat Dir der Jude Blut und Seele erst verdorben/ Dann bist du Deinem Volk und Vaterland gestorben. » $(31.10 .35$, I 227).

L'allocution mime le lien inter-personnel, qui apparaitt inversé dans l'emploi obligatoire de l'adresse au chef :

«Befehl, Hitler anzureden : Mein Führer ! » (10.8.34, I 134).

97 L'apostrophe est une figure de style qui sert à impliquer l'allocuté, à l'influencer: Chacun est appelé à se sentir concerné :

«Große Annonce in der Zeitung: Deutsche Männer und Frauen! Marschiert alle mit uns ! Kein Volksgenosse darf fehlen! » (16.2.34, I 88)

Klemperer constate la prise d'influence sur l'homme de la rue par la création de cette communauté du «wir», confronté à un seul «ich», le Führer. Ce sentiment d'appartenance se manifeste dans l'emploi du «wir» inclusif: «Es ist unsere Revolution» (9.7.33, I 39), « das plötzlich ganz offene Prahlen in Wort und Bild mit unseren Rüstungen » (6.12.38, I 441). Voici le discours d'un homme de la rue :

«Dieser Mann... äußert Gedanken, die in Form und Inhalt rein nationalsozialistisch sind. Von der Notwendigkeit der Volksgemeinschaft, von den für sich bestehenden Stämmen, von der Identität zwischen Recht und Macht, von der fraglosen Überlegenheit des neueun deutschen Heeres (denn wir wollen ja nicht angreifen und nur Frieden haben.)» (13.7.37, I 366)

Il arrive cependant au Führer d'utiliser le pluriel de majesté exclusif :

« Heute eine Hitlerrede in Würzburg, wieder mal klarer religiöser Wahnsinn. Nur daß er nicht ich, sondern wir sagte. 'Die Vorsehung führt uns, wir handeln dem Willen des Allmächtigen entsprechend' » (28.7.37, I 365). 
Ce lien personnel direct, qui est censé unir le peuple à son $\operatorname{chef}^{20}$, est donc aussi symbolisé par l'emploi du déterminatif personnel: Hitler dit: "Meine Soldaten» (4.1.42, I 6), lien inter-personnel qui apparaît inversé dans le passage suivant :

«Gestrige Schlagzeile der Dresdner NN : 'Das Volk der achtzig Millionen grüßt seinen großen Führer' » (28.10.38, I 425). "Neulich schickte mir Jelsky eine Predigt, die er einem verstorbenen Gemeindevorstand gehalten. Die Überschrift hieß 'Unserem Führer' » (4.7.34, I 123). Klemperer note avec mépris que certains juifs tentent ainsi de s'intégrer à la Volksgemeinschaft ! alors qu'ils sont exclus de cette communauté du wir.

\section{d) L'hypostase}

102 Klemperer relève, dès février 1935, l'emploi particulier des déterminatifs dans le langage de propagande :

103 «Der ostsächsische Mensch (contradictio in adjecto) - Der Mensch, der Student, die Hochschule etc, etc. » (21.2.35, I 184). « Auf dem Kongreß der Psychiater 'das nordische Kind'... eine große Inschrift auf Anschlagetafeln der Zeitung 'der Stürmer' : 'Die Juden sind unser Unglück... Wer den Juden kennt, kennt den Teufel' Usw.» (14.4.35, I 192). "In allen Ecken, in hundert Schaufenstern das ekelhafte Werbeplakat der 'politischen Wanderausstellung: Der ewige Jude'.» (4.4.39, I 467). « Bis zum letzten Wahnsinn die Konzentration des Hasses. Nicht England oder USA oder Rußland - nur, in allem nur und einzig der Jude. » (28.4.42, II 74). « Leitartikel der 'Dresdener Zeitung'... 'Juden in der Normandie' [...] da erschienen schon die 'ewig Gierigen', 'die Krummnasigen', um Besitz zu ergreifen [...] Wichtig an alledem ist mir dies : Die Judenhetze, das Zusammenziehen aller Feinde in den Feind, namens Jude [...] der Jude ist in jeder Hinsicht Zentralpunkt der LTI. » (20.7.44, II 547).

De tels emplois confèrent de l'existence à une ou à des entités fictives faussement considérées comme une réalité. L'emploi de der suppose l'extraction d'un individu parmi un ensemble. Il fait passer le nom de la langue à la parole en lui donnant le statut d'une expression référentielle. Les groupes nominaux ainsi introduits présupposent l'existence d'un référent, on en voit bien tout l'intérêt idéologique quand il s'agit de présenter sur le mode du « cela va de soi » « das Volk der Juden ». (17.12.41, I 695). En utilisant l'expression définie, le propagandiste fait comme si l'existence de l'objet correspondant ne faisait pas de doute. Il est plus difficile d'attaquer les posés que les présupposés.

105 En même temps, l'expression «der Jude », qui postule un existant, s'accompagne d'une indétermination référentielle qui empêche d'identifier un individu précis, de même, l'indétermination des individus impliqués par die dans die Juden renvoie au collectif d'un groupe nominal générique ${ }^{21}$. Klemperer commente cet emploi répétitif du déterminatif en d-, qu'il note aussi bien dans la langue des vainqueurs que dans celle des victimes, ainsi :

«der Jude, der Engländer - nichts als Kollektiva, kein Individuum mehr. Alter Sprachgebrauch erneuert, erweitert, überbetont, verweltanschaulicht. In der jüdischen Zeitung [...] steht oft und ekelerregend der jüdische Mensch [...]. Wann tritt eine Redewendung das erste Mal auf? Wann gewinnt sie allgemeine oder Epochenbedeutung? » (30.11.40, I 565).

106 L'emploi du défini désigne généralement un objet ou un être immédiatement identifiable dans la situation d'énonciation (ex. der Führer, der Jude Klemperer LTI, 85). 
Mais dans les exemples cités, il s'agit de nier toute spécificité individuelle, soit en extrayant une entité d'un ensemble dénombrable au singulier : der jüdische Mensch, ou bien encore (au pluriel), en visant l'ensemble dans sa totalité : die Krummnasigen, ou comme collectif : die internationale Judenkanaille.

La valeur générique s'établit ici, le plus souvent, par l'intermédiaire d'un membre de la classe représentatif de l'ensemble, qui isole pour mieux le stigmatiser, le juif qui s'oppose à der nordische Mensch. L'utilisation du générique pour désigner la classe en tant que concept (der) ou la classe définie en extension par les membres qui la composent (die) sert à accréditer l'idée, imputable à l'hypostase, de l'existence de caractéristiques substantielles, innées, der Jude: «plattfüssig, krummnasig, wasserscheu » (LTI, 190), donc à présupposer l'existence de caractéristiques raciales.

\section{e) Les Actes de langage}

108 Contredisant le pessimisme qui saisit parfois Klemperer sur la portée de la documentation qu'il a réunie :

«Als bloßes LTI würde mein vorschwebendes Opus wenig mehr enthalten als zwei Dutzend Wörter und Wendungen. Ich muß erweitern - aber wohin erweitern? » (9.6.42, II 117),

ses lecteurs d'aujourd'hui peuvent y découvrir des aspects du langage totalitaire qui permettent de comprendre ses mécanismes de communication et de manipulation. Ainsi en va-t-il de son aspect fonctionnel. On distingue en effet dans certains exemples relevés par Klemperer, des actes de langage ${ }^{22}$.

110 La rhétorique nazie utilise les actes de langage pour influer sur les croyances et l'action de ses destinataires, le peuple allemand ou les juifs. Le fascisme prétend imposer au travers du langage, sa réalité. Les actes de langage sont, dans une société démocratique, envisagés comme le produit d'une collaboration active, bien que parfois conflictuelle, entre les interlocuteurs. Or, ici les discours montrent une communication à sens unique constituée d'assertions ${ }^{23}$ péremptoires et programmatiques comme : «Adolph Hitler ist Deutschland » (11.9.34, I 143), « Die Jugend 'liebt die Eindeutigkeit und Entschlossenheit unserer Führung' [...] Deutsch sein heißt klar sein.» (11.9.34, I, 143), affirmations apodictiques, alors que manquent les assertions vraies, c'est-à-dire celles qui se soumettent à la contradiction.

111 Les actes de langage ne sont pas des éléments isolés, mais ils participent d'une stratégie, ils sont agencés en une construction hiérarchique destinée à produire certains effets. Certaines notations de Klemperer décrivent des actes de langage, comme dans le passage suivant :

«Gestern, als er (der Führer) die Auto-Ausstellung in Berlin eröffnete..., ging mir das Grundprinzip der gesamten Sprache des dritten Reichs auf : das böse Gewissen ; sein Dreiklang : sich verteidigen, sich rühmen, anklagen - niemals ein Moment des ruhigen Aussagens. » (19.2.38, I 397).

ou bien encore les montrent en acte :

« Führer, befiehl, wir folgen.» $(L T I, 93)$

On ne sera pas surpris que Klemperer s'attache surtout à relever l'acte de menace dans le journal :

« Neujahrsbotschaft Lutzes an die SA. (Februar 1938 - LTI 175). Die Reichstagsrede Hitlers wie eine Kriegsdrohung (verstärktes Heer). » (19.2.38, I 397), « Hitler droht 
mit Vernichtung der Juden in Europa, wenn 'sie' den Krieg gegen Deutschland heraufbeschwören würden. » (5.2.39. I, 461).

Parfois, la mise en garde précède la menace :

"vor allem aber: die große Warnung, nicht das "zersetzende Lügengift" der Auslandssender zu hören, mit den abschreckenden Urteilen : 2 1/2 Jahre Zuchthaus für eine Familie in Danzig. » (24.12.39, I 506).

Le peuple est sommé de choisir entre :

« Völkerfrieden oder Judendiktatur » (1.3.38, I 398-399)

116 Cependant, dans le langage de propagande nationalsocialiste, un acte de langage est totalement évacué, c'est la question, ceci est observé avec perspicacité par Klemperer qui désespère :

«Ich frage mich jetzt so oft nach Dingen (z.B. sprachlicher Natur), die mir fünfzig Jahre selbstverständlich waren. Hauptsache für die Tyrannis jeglicher Art ist das Unterdrücken des Fragetriebs. Und das ist so leicht zu machen. Wenn ich, Professor usw., lebenslang auf Denken geschult, mir so viele und so naheliegende Fragen durch fünfzig Jahre nicht gestellt habe, wie soll dann das Volk aufs Fragen kommen. Man braucht es eigentlich gar nicht zum Gegenteil erst anzuhalten.» (10.4.41, I 402).

117 Klemperer dépeint le fascisme comme une période où poser une question, même adressée à soi-même, est un acte tabou. Seules les questions rhétoriques subsistent (cf. Mein Kampf, ch. X, «Ursachen des Zusammenbruchs»), mais il s'agit alors de réponses déguisées en questions.

Parmi les actes de langage cités, certains relèvent d'actes indirects ${ }^{24}$. Ainsi en est-il de : « Du gibst dem Führer dein Ja.» (T1, 165), injonction sous couvert d'assertion. Klemperer en fournit l'illustration dans les exemples suivants :

« Der ständige Hinweis : 'Verräter werden vernichtet' » (4.9.39, I 485).

Cet énoncé est à la fois une information (Hinweis), une promesse (werden + infinitif) doublée d'une menace ${ }^{25}$.

« Die jüngste Drohung, von Estreicher (an Frau Voß) übermittelt, hieß : 'Wir können Sie binnen drei Tagen ins polnische Ghetto und in den Arbeitsdienst bringen.'» (9.11.41; I 685).

Ici l'information se double d'une menace. Dans l'exemple suivant, il s'agit d'un compterendu d'un acte de langage de menace :

«Drohung nach innen, z. T. fast mit gleichen Worten: Wer sabotiere oder die Autorität des Regimes antaste, gleichviel unter welcher Tarnung, der sterbe eines schimpflichen Todes. » (12.12.41, I 694).

121 Klemperer décèle donc parfaitement ce que les pragmalinguistes décrivent comme la combinaison de valeurs illocutoires : ainsi, Hitler présente au Reichstag sous la forme d'une requête (Bitte) une injonction doublée d'une menace :

«Hitler bittet den Reichstag um das neue Recht, 'weil es sich unter Millionen Anständiger um nur ganz wenige Ausnahmen handelt'. Damit wird aus der allgemeinen Forderung etwas Bestimmtes. In gewissem Sinn ist das enzyklopädischer Stil der Gewalt. Zugleich offene und versteckte Drohung. Sprachlich durchzudenken. » (28.4.42, II 75).

Bien sûr, on rencontre aussi des ordres directs ou indirects comme :

« Der Jude ist verpflichtet, seine Möbel (die er nicht verkaufen darf!) 'pfleglich' zu behandeln ». (19.4.42, II 69). 
Bizaremment, il manque dans ce relevé les promesses explicites, qui doivent être déduites d'énoncés fragmentaires, par exemple, à partir de slogans et des mots d'ordre comme :

«Blut und Boden», «Achse und Endsieg» (29.4.44, II 506-507), « für morgen 30. Januar (1944), den Tag der 'Machtübernahme' sind Versammlungen angekündigt unter der Parole : 'Dem deutschen Sieg entgegen'! » (27.1.44, II 481), ou encore « Heim ins Reich!» $(21.1 .40$, I 510)

Toute cette sphère de prise d'influence par l'utilisation du langage en acte est donc bien présente dans cette documentation.

\section{Conclusion}

S'intéresser au phénomène linguistique sous l'aspect du social et du politique, comme l'a fait Klemperer, c'est réfléchir à l'influence qu'on peut exercer sur les autres par l'emploi des mots. On reconnait maintenant, après la disparition d'autres didactures que celle du nazisme, que la réalité peut être suscitée et modifiée par «le pouvoir des mots $»^{26}$.

Par son relevé, au jour le jour, des pratiques langagières du nazisme, Klemperer œuvre à la fois en continuateur d'une Sprachkritik traditionnelle, mais aussi en précurseur d'une Sprachkritik actuelle, qui recherche les normes auxquelles se réfèrent ceux qui ont le pouvoir à partir de 1933 et dévoile les conflis de communication dans la société. Il est pour nous particulièrement important d'être témoins de la résistance d'un homme interdit de parole et d'écriture parce qu'il était juif. Klemperer montre bien à l'oeuvre une communication à sens unique, le langage des vainqueurs, auquel s'adaptent et sur lequel se calquent les vaincus :

«Es war immer, gedruckt und gesprochen, bei Gebildeten und Ungebildeten, dasselbe Klischee und dieselbe Tonart. Und sogar bei denen, die die schlimmsten verfolgten Opfer und mit Notwendigkeit die Todfeinde des Nationalsozialismus waren, sogar bei den Juden, herrschte überall, in ihren Gesprächen, in ihren Briefen, auch in ihren Büchern, solange sie publizieren durften, ebenso allmächtig wie armselig, und gerade durch ihre Armut allmächtig, die LTI. »

Klemperer montre que les clivages politiques ne se traduisent pas toujours par des clivages linguistiques, le langage entraîne, il est opaque, il permet à celui qui veut déguiser sa pensée de déguiser ses mots. En effet, comme le démontre Hitler, employer le terme «Friede» n'est pas l'apanage des pacifistes. Pour résister, il faut d'abord percevoir.

L'utilisation du langage à des fins purement idéologiques se distingue de l'usage ordinaire des sociétés fondées sur le consensus. Quand nous parlons, nous sommes contraints d'utiliser les catégories temporelles ou spatiales, mais nous avons la possibilité de nous soustraire au langage idéologique et de le refuser. C'est bien ce que Klemperer a fait.

Le monopole de la communication dans les mains d'un pouvoir totalitaire livre le peuple à la propagande et à son interprétation tendancieuse de la réalité, Klemperer lutte avec LTI contre le pouvoir de la propagande. Mais, de surcroît, il conduit à s'interroger sur le pouvoir des mots et des actes de langage dans une société moderne technicienne qui a tendance à oublier l'humain. LTI stigmatise une communication à sens unique, la perversion du dialogue, forme typique de la démocratie. 

l'uniformité de la LTI, prison de langage qui englobe vainqueurs et victimes, Klemperer illustre comment une société peut faire violence à l'individu et au peuple à travers ses mots.

Ainsi que le remarque G. Stötzel $(1981,289)$ :

« Das Prinzip der Freiheit im gesellschaftlichen Handeln kann also realisiert werden durch die Erscheinung unterschiedlichen, konkurrierenden Sprachgebrauchs. »

\section{BIBLIOGRAPHIE}

Ehlich Konrad (éd.), 1989 : Sprache im Faschismus. Suhrkamp Taschenbuch. Frankfurt/Main. Fernandez-Bravo Nicole, 1996 : « 1945 - Le réveil d'une attitude critique vis-à-vis du langage et du pouvoir des mots ». In : Höpel Thomas, Tiemann Dieter (éd.), 1945-1950 Jahre danach. Aspekte und Perspektiven im deutsch-französischen Beziehungsfeld. Leipziger Universitätsverlag.

Haug Wolfgang Fritz, 1968 : « Der hilflose Antifaschismus. ». In Heringer H. J. (éd.) (1988), 150-161.

Heringer Hans Jürgen (éd.), 1981, 1988 (2. Auflage) : Holzfeuer im hölzernen Ofen. Aufsätze zur politischen Sprachkritik. Gunter Narr Verlag. Tübingen.

Kämper Heidrun, 1996 : « Zeitgeschichte - Sprachgeschichte. Gedanken bei der Lektüre des Tagebuchs eines Philologen. Über die Ausgaben von Victor Klemperers Tagebuch 1933-1945 ». In : ZGL, 24, 328-341.

Kerbrat-Orecchioni Catherine, 1977 : La connotation. Presses universitaires de Lyon.

Klaus Georg, 1964 : Die Macht des Wortes. Ein erkennnistheoretisches Traktat. Berlin Ost. 1972 (6. Auflage).

Klemperer Victor, 1993 : LTI. Aus dem Notizbuch eines Philologen. 12. Auflage. Reclam. 1996 : «Ich will Zeugnis ablegen bis zum letzten». Tagebücher 1933-1941 (Band 1), Tagebücher 1942-1945 (Band 2). 8. Auflage. Aufbau Verlag.

Klein Josef (éd.), 1989 : Politische Semantik. Bedeutungsanalytische und sprachkritische Beiträge zur politischen Sprachverwendung. Westdeutscher Verlag.

Plett Heinrich F. 1985 : Einführung in die rhetorische Textanalyse. 6. Auflage. Helmut Buske. Hamburg.

Lübbe Hermann, 1988 : « Der Streit um Worte, Sprache und Politik ». In : Heringer H.J. éd. 1988, 48-70.

Sternberger D., Storz G., Süskind E., 1957 : Aus dem Wörterbuch des Unmenschen. HamburgDüsseldorf. 1968, avec le sous-titre : Neue erweiterte Ausgabe mit Zeugnissen über die Sprachkritik. Polenz Peter von, 1967 : « Sprachpurismus und Nationalsozialismus. Die 'Fremdwort'-Frage gestern und heute ». In : Germanistik - eine deutsche Wissenschaft. Suhrkamp, 111-165. 
Stötzel Georg, 1995 : « Der Nazi-Komplex ». In: Stötzel G., Wengler M. : Kontroverse Begriffe. Geschichte des öffentlichen Sprachgebrauchs in der Bundesrepublik Deutschland. De Gruyter, 355-383.

Maas Utz : «Sprache im Nationalsozialismus. Analyse einer Rede eines Studentenfunktionärs ». In : Ehlich K. (éd.) (1989), 162-198.

Sauer Wolfgang Werner : « Der Duden im ‘Dritten Reich’ ». In : Ehlich K. (éd.) (1989), 104-120.

Winckler Lutz, 1970 : Studie zur gesellschaftlichen Funktion faschistischer Sprache. Suhrkamp.

\section{ANNEXES}

Aus dem Wörterbuch des Unmenschen :

Anliegen - Auftrag - Ausrichtung - Bedeutung - charakterlich - durchführen - echt/ einmalig - Einsatz - erarbeiten - Frauenarbeit - Gestaltung - Härte - herausstellen intellektuell - Kontakte - Kulturschaffende - Lager - leistungsmäßig - Menschen Menschenbehandlung - organisieren - Problem - Propaganda - querschießen - Raum Ressentiment - Schulung - Sektor - tragbar -untragbar - Vertreter - Verwendung Wissen um - Zeitgeschehen.

(Les termes qui figurent aussi chez Klemperer sont marqués en gras.)

L'extrait du journal du 24.4.45 II, 754 présente un florilège des phénomènes décrits dans cet article :

« Gestern nachmittag kam der alte Tyroller aufgeregt, 'sie' seien schon bei Gabelingen, dem Flugplatz dicht vor Augsburg, und bei Dillingen seien sie auf unzerstörter Brücke über die Donau gegangen [...]

Wir sahen in der Zeitung den 'Tagesbefehl des Führers an die Ostfront'. Bis zuletzt diesselben Züge der Sprache und des Denkens. Maßlose Beschimpfung und Verleumdung des Gegners. 'Der jüdisch-bolchevistische Todfeind' ist 'zum letzten Mal' zum Angriff angetreten. (Doppeldeutigkeit des 'letzten Mals'.) Er will uns 'ausrotten', 'während die alten Männer und Kinder ermordet werden, werden Frauen und Mädchen zu Kasernenhuren erniedrigt. Der Rest marschiert nach Sibirien. 'Aber wir haben alles vorausgesehen und unser Heer 'durch zahllose neue Einheiten ergänzt.' (Verlogener Superlativ.) Aber : 'Der Bolchevismus wird dieses Mal das alte Schicksal Asiens erleben, d.h. er muß und wird vor der Hauptstadt des deutschen Reiches verbluten.' Nachher noch einmal, das deutsche Volk hoffe, daß 'durch euren Fanatismus'.... der bolchevistische Ansturm in einem Blutbad erstickt.' Wieder die Vorspiegelung einer Möglichkeit des Sieges : 'Im Augenblick, in dem das Schicksal den größten Kriegsverbrecher aller Zeiten von dieser Erde genommen hat, wird sich die Wende dieses Krieges entscheiden.' - Rhetorisch formuliert : 'Berlin bleibt deutsch, Wien wird wieder deutsch, und Europa wird niemals russisch.' LTI. »

\section{NOTES}

1. . Cf. «Wissenschaft, die sich mit Texten historischen, literarischen oder kulturhistorischen Inhalts in einer bestimmten Sprache beschäftigt und sie sprachlich, historisch, 
kulturgeschichtlich und gesellschaftlich interpretiert » (R. Klappenbach/W. Steinitz (1975) : « Wörterbuch der deutschen Gegenwartssprache, 4. band, 2790), Akademie-Verlag. Berlin.

2. . Cf. les chroniques linguistiques qui paraissent de 1945 à 1948 dans la revue mensuelle die Wandlung signées par Sternberger, Süskind, Storz, réunies en ouvrage en 1957 sous le titre : Aus dem Wörterbuch des Unmenschen.

3. . E. Benvéniste (1965): Problèmes de linguistique générale. Gallimard.

4. . «LTI, LQI. Von der Unschuld der Sprache und der Schuld der Sprechenden », titre de la communication de K. Ehlich au Congrès de l'IDS à Mannheim: Das 20. Jahrhundert. Sprachgeschichte - Zeitgeschichte, le 13 mars 1997.

5. . Voir annexe.

6. . Cf. W. W. Sauer $(1989,109)$ indique qu'une grande partie du vocabulaire national-socialiste est déjà constitué en 1933 et fonctionne donc comme instrument de conquête du pouvoir.

7. . « Auf der einen Seite sind es die Formen, die unter den Begriffen Sprache der verwalteten Welt, Sprache der rationalisierten Welt, Organisationssprache oder Institutionssprache bekannt geworden sind, auf der anderen Seite die des ideologischen Sprechens, der Meinungs- und Propagandasprache. Wir unterscheiden [...] Funktionssprache und Meinungssprache. Die Funktionssprache dient der organisatorischen Verständigung innerhalb des staatlichen Apparates und seiner Institutionen. In ihr wird der gesamte institutionelle und fachsprachliche Wortschatz der Politik aktualisiert. Sie ist wirklichkeitsbezogen und hat ein stark rationales Gepräge. Die Meinungssprache vermittelt die Deutungen, die die Ideologie von der Wirklichkeit gibt und richtet sich nach außen an die Öffentlickeit. Sie enthält einen starken Einschlag ideologischen Vokabulars ».

8. . Cité in : Ehlich 1989, 112.

9. Cf. la définition de Kerbrat-Orecchioni $(1977,215)$ : «Il ne nous semble pas que la connotation puisse être assimilée à l'idéologie..., étant entendu que par 'idéologie' nous entendons : un système de représentations... de nature interprétative... jouant un rôle historique et politique précis... qui tend fallacieusement à s'universaliser et se naturaliser... et qui, tout en investissant le langage verbal, constitue elle-même un langage autonome.» Utz Maas (1989, donne la définition suivante: «Konnotation meint..., daß jeder sprachliche Ausdruck in unserer Sprachbiographie durch den Kontext indiziert ist, in dem wir ihn kennengelernt haben. »

10. . Sauer $(1989,106)$ note qu'il fait son apparition dans Duden 12 (1934).

11. . Organisationen, Institutionen, Menschen zwangsweise der nazistischen Ideologie angleichen, unterwerfen. (Klappenbach/Seinitz. t. 3, 1604).

12. . Cf. v. Polenz $(1967,135)$.

13. Voici encore quelques exemples de son abandon aux métaphores biologiques : « Die LTI ist von der teutschen (Romantik) tyrannisch regiert, aber nicht so, daß die deutsche ausgeschaltet, sondern so, daß sie [...] nur eben von der teutschen verseucht wird.» (23.6. - 1.7.41, I 623). Il évoque dans les mêmes termes biologiques das Gift, die Seuche am deutschen Volkskörper: Die Seuche wütet in allen, vielleicht ist es nicht Seuche, sondern deutsche Grundnatur » (25.10.41, I 681) ou encore «Deutschland... ganz und in allem undeutsch... Nur in der Blutidee nicht, im Animalischen also. » (12.9.34 I 143, 144).

14. . C'est moi qui indique en italique les termes marqués.

15. . In : Deutsche Sprache im 20. Jahrhundert. (1978), 66, 2. Auflage. Pipper.

16. . In: Tendenzen in der Gegenwartssprache. Sprachvarietäten. (1993), 236, 3. Auflage. Kohlhammer.

17. . «(Der Gegenstand) wird vergrößert [...]. Die dadurch bewirkte Überbewertung der Wirklichkeit löst den dargestellten Gegenstand aus seinen lebensweltlichen Bezügen und versetzt ihn in eine imaginäre Vorstellungswelt. » (Plett 1985, 76).

18. . Cf. Houston Chamberlain. Seite 817. 
19. . Cf. Klein $(1989,11)$ : «Als 'politische Schlagwörter' werden Wörter dann bezeichnet, wenn sie in öffentlichen Auseinandersetzungen häufig, oft inflatorisch verwendet werden und wenn sie in komprimierter Form politische Einstellungen ausdrücken oder proponieren. Schlagwörter dienen als Instrument der politischen Beeinflussung. Mit ihnen wird versucht, Denken, Gefühle und Verhalten zu steuern, soweit sie politisch relevant sind. Sie sind eine Hauptwaffe der politischen Auseinandersetzung. "

20. . Les adresses des lettres au Führer montrent souvent un caractère dythirambique comme celle-ci (Sommer 1939 Victoria v. Dirksen) «Mein so sehr geliebter, fabelhafter beinah unwahrscheinlicher Führer » In : Die Rückseite des Hakenkreuzes. (1995) DTV Dokumente Nr. 2967.

21. « La généricité nominale exige, en plus de la non-spécificité, d'une part, une extension non partitive (classe, ensemble etc.) et, d'autre part, une identification non spécifique » (G. Kleiber, H. Lazarro, 1987, cité in Kleiber Anaphores et pronoms. (1984) Duculot, 171).

22. C'est-à-dire des actes accomplis au moyen d'énoncés. Un acte de langage c'est une suite linguistique, une séquence qui prétend agir sur les destinataires et les faire agir.

23. . L'assertion prétend dire le vrai. Il s'agit cependant ici de jugements (Behauptungen) sujets à caution comme : « Du bist nichts, dein Volk ist alles ». (LTI, 24).

24. . Ils ont une intention de communication différente de celles qu'ils affichent. Un énoncé peut, en effet associer plusieurs valeurs d'actes.

25. . On promet à l'interpelé d'agir de manière défavorable à son égard.

26. . Die Macht des Wortes, titre du livre de Georg Klaus (1964).

\section{RÉSUMÉS}

Cet article étudie quelques caractéristiques du langage de propagande sous le nationalsocialisme, dont le vocabulaire significatif est commenté par Klemperer dans son journal intime, et dénommé par lui LTI (Lingua Tertii Imperii).

Dans l'optique d'une Sprachkritik actuelle, qui voit dans ce langage une variété d'emploi, le lexique idéologique national-socialiste, relevé par Klemperer, avec ses connotations, ses euphémismes, ses mots "dans l'air du temps", ses mots savants, ainsi que le langage institutionnel et ses néologismes, font l'objet d'analyses. D'autre part, les aspects rhétoriques de ce langage de propagande y sont décrits dans la perspective de la pragmatique linguistique et de la théorie de l'argumentation. Klemperer apparaît dans son Journal comme un homme sensible au pouvoir des mots dans le langage quotidien et qui fait œuvre de « résistance» en montrant l'uniformité de la LTI, prison de langage qui enferme « vainqueurs » et victimes.

Es geht Klemperer in seinem Tagebuch darum, die Herrschaft durch Sprache zur Zeit des Nationalsozialismus $\mathrm{zu}$ dokumentieren. Klemperer erkannte in den herausgegriffenen « Ausdrücken » das ideologisch geprägte Vokabular der Meinungssprache und die Funktionssprache der NS-Propaganda. Er wollte durch die Untersuchung der sogenannten LTI (Lingua Tertii Imperii) Erkenntnisse über die Wirkung des Faschismus gewinnen. Im Artikel geht es darum, das von Klemperer aufgegriffene und für die nationalsozialistische Verwendung typische Vokabular mit den Mitteln der linguistischen Pragmatik, der Rhetorik und der Argumentationstheorie zu untersuchen. Klemperer zeigt besonders eindringlich die ideologische Verstrickung der Menschen in die Propagandasprache, wenn sie kaum Möglichkeiten haben, sich ihr zu entziehen. 
AUTEUR

NICOLE FERNANDEZ BRAVO

Université Paris X Nanterre 